\title{
Dynamics of ripple formation in sputter erosion: nonlinear phenomena
}

\author{
S. Park ${ }^{1}$, B. Kahng ${ }^{1,2}$, H. Jeong ${ }^{2}$, and A.-L. Barabási ${ }^{2}$ \\ ${ }^{1}$ Department of Physics and Center for Advanced Materials and Devices, Konkuk University, Seoul 143-701, Korea \\ ${ }^{2}$ Department of Physics, University of Notre Dame, Notre Dame, IN 46556
}

\begin{abstract}
Many morphological features of sputter eroded surfaces are determined by the balance between ion induced linear instability and surface diffusion. However, the impact of the nonlinear terms on the morphology is less understood. We demonstrate that while at short times ripple formation is described by the linear theory, after a characteristic time the nonlinear terms determine the surface morphology by either destroying the ripples, or generating a new rotated ripple structure. We show that the morphological transitions induced by the nonlinear effects can be detected by monitoring the surface width and the erosion velocity.
\end{abstract}

PACS numbers:68.55.-a,05.45.+b,64.60.Cn,79.20.Rf

The morphological evolution of ion sputtered surfaces has generated much experimental and theoretical interest in recent years. As a result, there is extensive evidence that ion bombardment can result in ordered surface ripples, or lead to kinetic roughening, depending on the experimental conditions. These experimental results, which cover amorphous and crystalline materials $\left(\mathrm{SiO}_{2}\right.$ [1]), and both metals (Ag [2]) and semiconductors (Ge [3], Si [4.5]), have motivated extensive theoretical work aiming to uncover the mechanism responsible for ripple formation and kinetic roughening. A particularly successful model has been proposed by Bradley and Harper (BH) [6], in which the height $h(x, y, t)$ of the eroded surface is described by the linear equation

$$
\partial_{t} h=\nu_{x} \partial_{x}^{2} h+\nu_{y} \partial_{y}^{2} h-K \partial^{4} h,
$$

where $K$ is the surface diffusion constant and the coefficients $\nu_{x}$ and $\nu_{y}$ are induced by the erosion process such that $\nu_{y}<0$ and $\nu_{x}$ can change sign as the angle of the incidence of the ions is varied. The balance of the unstable erosion term $\left(-|\nu| \partial^{2} h\right)$ and the smoothening surface diffusion term $\left(-K \partial^{4} h\right)$ generates ripples with wavelength

$$
\ell_{i}=2 \pi \sqrt{2 K /\left|\nu_{i}\right|}
$$

where $i$ refers to the direction ( $x$ or $y$ ) along which the associated $\nu_{i}\left(\nu_{x}\right.$ or $\left.\nu_{y}\right)$ is the largest. While successful in predicting the ripple wavelength and orientation [7], this linear theory cannot explain a number of experimental features, such as the saturation of the ripple amplitude [8 10], the observation of rotated ripples [1], and the appearance of kinetic roughening 12,13. Recently it has been proposed 14 that the inclusion of nonlinear terms and noise (both of which were derived from Sigmund's theory of sputtering 115]) can cure these shortcomings. Consequently, Eq.(1) has to be replaced by the noisy Kuramoto-Sivashinsky equation (KS) equation [16]

$$
\partial_{t} h=\nu_{x} \partial_{x}^{2} h+\nu_{y} \partial_{y}^{2} h-K_{x} \partial_{x}^{4} h-K_{y} \partial_{y}^{4} h-K_{x y} \partial_{x}^{2} \partial_{y}^{2} h
$$

$$
+\frac{\lambda_{x}}{2}\left(\partial_{x} h\right)^{2}+\frac{\lambda_{y}}{2}\left(\partial_{y} h\right)^{2}+\eta(x, y, t),
$$

where $\eta(x, y, t)$ is an uncorrelated white noise with zero mean, mimicking the randomness resulting from the stochastic nature of ion arrival to the surface. All coefficients in Eq.(3) have been determined in terms of the experimental parameters, such as the ion flux, angle of incidence, ion penetration depth, and substrate temperature [14, 17.

While it is expected that the nonlinear theory incorporates most features of ripple formation and kinetic roughening, the morphological and dynamical features of the surface described by it are known only in certain special cases. For example, when the nonlinear terms and the noise are neglected $\left(\lambda_{x}=\lambda_{y}=0, \eta=0\right)$, Eq.(3) reduces to the linear theory (1), and predicts ripple formation. It is also known that the isotropic KS equation $\left(\nu_{x}=\nu_{y}<0, K_{x}=K_{y}=K_{x y} / 2\right.$, and $\left.\lambda_{x}=\lambda_{y}\right)$ asymptotically (for large time and length scales) predicts kinetic roughening, with exponents similar to that seen experimentally in ion sputtering [12]. For positive $\nu_{x}$ and $\nu_{y}$, Eq.(3) reduces to the anisotropic KPZ equation [18], whose scaling behavior is controlled by the sign of $\lambda_{x} \cdot \lambda_{y}$ [19]. Finally, recent integration by Rost and Krug 20] of the noiseless version of Eq.(3) provided evidence that when $\lambda_{x} \cdot \lambda_{y}<0$, new ripples, unaccounted for by the linear theory, appear and their direction is rotated with respect to the ion direction [20]. However, it is not known if this rotated phase survives in the presence of noise, $\eta$. These special cases illustrate the complexity of the morphological evolution predicted by Eq.(3). To be able to make specific predictions on the morphology of ion-sputteted surfaces, we need to gain a full understanding of the behavior predicted by (3), going beyond the special cases, that are often experimentally irrelevant. In this paper, we integrate numerically Eq.(3), aiming to uncover the dynamics and the morphology of the surfaces 
for different values of the parameters. We demonstrate a clear separation of the linear and nonlinear behavior. For short erosion times, the ripple development and orientation follow the predictions of the linear theory of $\mathrm{BH}$. However, after a well defined crossover time, which depends on the coefficients of Eq.(3), the surface morphology is determined by the nonlinear terms. We find that when $\lambda_{x} \cdot \lambda_{y}>0$ the nonlinear terms destroy the ripple morphology. However, when $\lambda_{x} \cdot \lambda_{y}<0$, they result in a long and apparently rough transient regime, followed by a new morphology of rotated ripples, as seen in the noiseless KS equation. We show that these morphological transitions can be detected by monitoring the surface width or erosion velocity, quantities that can be measured more easily in situ. Finally, we discuss the impact of our result on current and future experimental work.

The direct numerical integration is carried out by discretizing the continuum equation of (3), using the standard discretization techniques [21]. We choose a temporal increment $\Delta t=0.01$ and impose periodic boundary conditions $h(x, y, t)=h(x+L, y, t)=h(x, y+L, t)$ where $L \times L$ is the size of the substrate. We choose the noise to be uniformly distributed between $[-1 / 2,1 / 2]$, and perturb the initial flat configuration with the noise. Since the sign of the nonlinear terms plays a significant role in defining the surface morphology, we discuss separately the $\lambda_{x} \cdot \lambda_{y}>0$ and $\lambda_{x} \cdot \lambda_{y}<0$ cases.

$\lambda_{x} \cdot \lambda_{y}>0$ - A general feature of sytems such as Eq.(3) is that the nonlinear terms do not affect the surface morphology or dynamics until a crossover time $\tau$ has been reached. Thus, we expect that for early times, i.e. for $t<\tau$, the surface morphology and dynamics is properly described by the linear theory. To demonstrate this separation of the linear and nonlinear regimes, in Fig. 1 we show the time dependence of the surface width defined as $W^{2}(L, t) \equiv \frac{1}{L^{2}} \sum_{x, y} h^{2}(x, y, t)-\bar{h}^{2}$ and of the mean height $\bar{h}=\frac{1}{L^{2}} \sum_{x, y} h(x, y, t)$. We find that for $t<\tau$, the width $W$ increases exponentially while the mean height stays constant at $\bar{h}=0$. Indeed, both of these findings are consistent with the predictions of the linear theory: $W$, being proportional to the ripple amplitude, according to Eq.(1) increases as $W \sim \exp \left(\nu t / \ell^{2}\right)$, and the linear terms do not change the mean height of the surface. Furthermore, inspecting the surface morphology, we find that in this regime the ripple wavelength and orientation are also correctly described by the linear theory. For example, for the parameters $\nu_{x}=-0.0001, \nu_{y}=-0.6169$, $K_{x}=K_{y}=K_{x y} / 2=2$, and $\lambda_{x}=\lambda_{y}=-0.001$, according to $(2)$, the ripple wavelength along the $y$ axis is $\ell_{y} \approx 16$, and along the $x$-axis is $\ell_{x} \approx 1257$. Since the dominant wavelength is determined by the growth rate $\sim \exp \left(\nu t / \ell^{2}\right)$, the smaller wavelength, i.e. $\ell_{x}$, will dominate. As Fig. 2a shows, for a system of size $64 \times 64$ we observe four ripples aligned along the $x$-axis, in agree- ment with the previous prediction. As a second example we consider the case, $\nu_{x}=-1.2337, \nu_{y}=-0.0001$, $K_{x}=K_{y}=K_{x y} / 2=1$, and $\lambda_{x}=\lambda_{y}=-0.001$, for which we expect ripples of wavelength $\ell_{x} \approx 8$, smaller than $\ell_{y} \approx 889$. As Fig. 2 b shows, in this case we observe eight ripples aligned along the $y$-axis.

While the early time behavior is correctly predicted by the linear theory, beyond the crossover time $\tau$ the nonlinear terms become effective. One of the most striking consequence of these terms is that the surface width stabilizes rather abruptly (see Fig. 1). Furthermore, the ripple pattern generated in the linear regime disappears, and the surface exhibits kinetic roughening. A typical surface morphology, demonstrating the absence of ripples, is shown in Fig. 2c. The crossover time $\tau$ from the linear to the nonlinear behavior can be estimated by comparing the strength of the linear term with that of the nonlinear term. Let the typical height at the crossover time $\tau$ be $W_{0} \equiv \sqrt{W^{2}(L, \tau)}$. Then, from the linear equation we obtain $W_{0} \sim \exp \left(\nu \tau / \ell^{2}\right)$, while from $\partial_{t} h \sim \lambda(\partial h)^{2}$ we estimate $W_{0} / \tau \sim \lambda W_{0}^{2} / \ell^{2}$. Combining these two relations we obtain

$$
\tau \sim\left(K / \nu^{2}\right) \ln (\nu / \lambda)
$$

In this expression, $\nu, K$ and $\lambda$, refer to the direction perpendicular to the ripple orientation. The predicted $\lambda$-dependence of $\tau$ is confirmed in the inset of Fig. 1a. An another quantity that refects the transition from the linear to the nonlinear regime is the erosion velocity $v=\partial_{t} \bar{h}$. The main contribution to the erosion velocity comes from a constant erosion rate $-v_{0}$, that has been omitted from (1) and (3), since it does not affect the surface morphology 6, 14]. However, in addition to $v_{0}$, the mean height is also modified by the nonlinear terms, $\lambda_{x}\left(\partial_{x} h\right)^{2}$ and $\lambda_{y}\left(\partial_{y} h\right)^{2}$. In the following for simplicity, we neglect the $v_{0}$ term, since its value does not depend on the surface morphology, and it is constant throughout the erosion process. The nonlinear terms act to decrease the mean height in the case of $\lambda_{x}<0$ and $\lambda_{y}<0$. We can estimate the surface velocity as $v \sim \lambda W_{0}^{2} / \ell^{2} \sim \nu^{3} /(K \lambda)$ using $W_{0} \sim \nu / \lambda$. This dependence of $v$ on $\lambda$ is consistent with the numerical results, shown in the inset of Fig. 1b. In this regime $(t>\tau)$ the surface exhibits kinetic roughening, i.e. the surface width should increase either logarithmically (when $\lambda=0$ ) or as a power law (when $\lambda \neq 0$ ) [19,22]. However, compared with the exponential increase in the early regime $(t<\tau)$, this dependence is hardly observable. The simulation times required to investigate the asymptotic scalings of $W$ with $t$ are currently prohibitive.

$\lambda_{x} \cdot \lambda_{y}<0-$ As Fig. 3a shows, we again observe a separation of the linear and nonlinear regimes, however, we find that the morphology and the dynamics of the surface in the nonlinear regime is quite different from the case $\lambda_{x} \cdot \lambda_{y}>0$. In regime I, for early times $(t<\tau)$, the surface forms ripples (see Fig. 4a), whose wavelength 
and orientation is correctly described by the linear theory. After the first crossover time $\tau$, given by Eq. (4), the surface width is stabilized, and the ripples disappear, as shown in Fig. 4b. After $\tau$, the system enters a rather long transient regime, that we call the regime II. Here, the surface is rough, and no apparent spatial order is present. We often observe the development of individual ripples, but they soon disappear, and no long-range order is present in the system. However, at a second crossover time $\tau_{2}$, a new ripple structure suddenly forms, as shown in Fig. 4c, in which the ripples are stable and rotated with an angle $\theta_{c}$ to the $x$ direction. The angle $\theta_{c}$ has the value $\theta_{c}=\tan ^{-1} \sqrt{-\lambda_{x} / \lambda_{y}}$, (or $\left.\tan ^{-1} \sqrt{-\lambda_{y} / \lambda_{x}}\right)$ [20], which can be calculated by moving to a rotated frame of coordinates that vanishes the nonlinear term in the transverse direction. Indeed, as Fig. 4c shows, the observed angle is in excellent agreement with $\theta_{c}=\tan ^{-1}(1 / 2)$ for $\lambda_{x}=1$ and $\lambda_{y}=-4$. We also find that the time the system spends in regime II fluctuates from system to system, thus $\tau_{2}$ has a wide distribution. The transitions between the three regimes can be detected by monitoring the surface width $W$ (Fig. 3a): in regime I, the width increases exponentially, as predicted by the linear theory; it is approximately constant but hightly fluctuating in regime II, and suddenly increases and stabilizes in regime III. Note that the amplitude of the rotated ripples in regime III is much larger than in regime II, a rather attractive feature for possible applications as patterned templates for various microelectronic applications.

The demonstrated morphological transitions generate an anomalous behavior in $\bar{h}$ as well. As Fig. 3a shows, the mean height is zero in the linear regime, increases as the ripples are destroyed in regime II, and decreases with a constant velocity in regime III. In order to understand this complex behavior, we consider a specific example, for which the surface morphologies are shown in Fig. 4. For this parameter set, ripples are aligned along the $y$-axis in the region I, because $\ell_{x} \ll \ell_{y}$. Thus, the contribution of $\left(\partial_{x} h\right)^{2}$ is much larger than that of $\left(\partial_{y} h\right)^{2}$, even though $\left|\lambda_{x}\right|<\left|\lambda_{y}\right|$, and the surface height increases due to the term $\lambda_{x}\left(\partial_{x} h\right)^{2}$ with $\lambda_{x}>0$ in regime II. However, as the ripples are destroyed by the nonlinear effects, the contribution of $\left(\partial_{y} h\right)^{2}$ term increases, and eventually $\lambda_{y}\left(\partial_{y} h\right)^{2}$ becomes larger than $\lambda_{x}\left(\partial_{x} h\right)^{2}$, forcing the mean height to decrease because $\lambda_{y}<0$. The velocity in regime III is determined by the nonlinear coefficient in the direction along the ripples, which reduces to $\lambda_{x}+\lambda_{y}$ after the coordinate transformation to the rotated ripple direction. This prediction is in good agreement with the results of Fig. 4, which demonstrates that $v \sim 1 /\left(\lambda_{x}+\lambda_{y}\right)$.

The clear separation of the linear and the nonlinear behavior, that holds for both signs of $\lambda_{x} \cdot \lambda_{y}$, has a direct impact on the experimental observations. Numerous experiments have observed the development of ripples whose wavelength and orientation is in good agreement with the prediction of the linear theory [1] 3, 5, ]1. Based on our results, we expect that these experiments were in the $t<\tau$ regime, where indeed the linear theory fully describes the system. However, recent results have provided detailed experimental evidence of ripple amplitude stabilization [8 10], a clear sign of the presence of nonlinear effects. Furthermore, it was found that the different $W$ versus $t$ curves can be collapsed by rescaling time with a factor $\nu^{2} / K$ and amplitude with $\sqrt{\nu / 2 K}$ 9]. Indeed, this is in excellent agreement with our prediction, Eq.(4). Finally, since the values of $\nu$ and $\lambda$ can be tuned by changing the ion energy and the angle of incidence, and $K$ can be tuned with the temperature, the values of $\tau$ and $\tau_{2}$ can be changed continuously, and thus our predictions on the morphological transition between the linear and nonlinear regimes could be tested experimentally. Furthermore, the detailed morphological evolution uncovered here, combined with earlier calculations that connect the coefficients in Eq.(3) to the numerical values of the parameters describing the ion-bombardment process [6,14, 17], offer a detailed roadway that can guide further experiments and facilitate the use of ion sputtering for surface patternings.

This work is supported by KOSEF (Grant No. 9710207-025-2), the Korean Research Foundation (Grant No. 98-015-D00090), NSF-DMR and ONR.

[1] T.M. Mayer, E. Chason, A.J. Howard, J. Appl. Phys. 76, 1633 (1994).

[2] S. Rusponi, C. Boragno, and U. Valbusa, Phys. Rev. Lett. 78, 2795 (1997).

[3] E. Chason, T.M. Mayer, B.K. Kellerman, D.T. McIlroy, and A.J. Howard, Phys. Rev. Lett. 72, 3040 (1994).

[4] G. Carter and V. Vishnyakov, Phys. Rev. B 54, 17647 (1996); Z.X. Jiang and P.F.A. Alkemade, Appl. Phys. Lett. 73, 315 (1998).

[5] J. Erlebacher, M.J. Aziz, E. Chason, M.B. Sinclair, and J.A. Floro, Phys. Rev. Lett. 82, 2330 (1999).

[6] R.M. Bradley and J.M.E. Harper, J. Vac. Sci. Technol. A 6, 2390 (1988).

[7] I. Koponen, M. Hautala, and O.-P. Sievaenen, Phys. Rev. Lett. 78, 2612 (1997).

[8] K. Wittmaack, J. Vac. Sci. Technol. A 8, 2246 (1990).

[9] J. Erlebacher, M.J. Aziz, E. Chason, M.B. Sinclair, and J.A. Floro, (preprint).

[10] J.J. Vajo, R.E. Doty, and E.-H. Cirlin, J. Vac. Sci. Technol. A 6, 76 (1988).

[11] S. Rusponi, G. Costantini, C. Boragno, and U. Valbusa, Phys. Rev. Lett. 81, 2735 (1998).

[12] E.A. Eklund, R. Bruinsma, J. Ruidnick, and R.S. Williams, Phys. Rev. Lett. 67, 1759 (1991).

[13] H.-N. Yang, G.-C. Wang, and T.-M. Lu, Phys. Rev. B 50. 7635 (1994).

[14] R. Cuerno and A.-L. Barabási, Phys. Rev. Lett. 74, 4746 
(1995).

[15] P. Sigmund, Phys. Rev. 184, 383 (1969).

[16] Y. Kuramoto, Chemical Oscillations, Waves, and Turbulence (Spinger, Berlin, 1984); G.I. Sivashinsky, Acta Astron. 4, 1177 (1977); G.I. Sivashinsky and D.M. Michelson, Prog. Theor. Phys. 63, 2112 (1980).

[17] M.A. Makeev and A.-L. Barabási, Appl. Phys. Lett. 71, 2800 (1997).

[18] M. Kardar, G. Parisi, and Y.-C. Zhang, Phys. Rev. Lett. 56, 889 (1986).

[19] D.E. Wolf, Phys. Rev. Lett. 67, 1783 (1991).

[20] M. Rost and J. Krug, Phys. Rev. Lett. 75, 3894 (1995).

[21] W.H. Press, B.P. Flannery, S.A. Teukolsky, and W.T. Vetterling, Numerical Recipes (Cambridge Univ. Press, Cambridge, 1986).

[22] A.-L. Barabási and H.E. Stanley, Fractal Concepts in Surface Growth (Cambridge University Press, Cambridge, 1995).
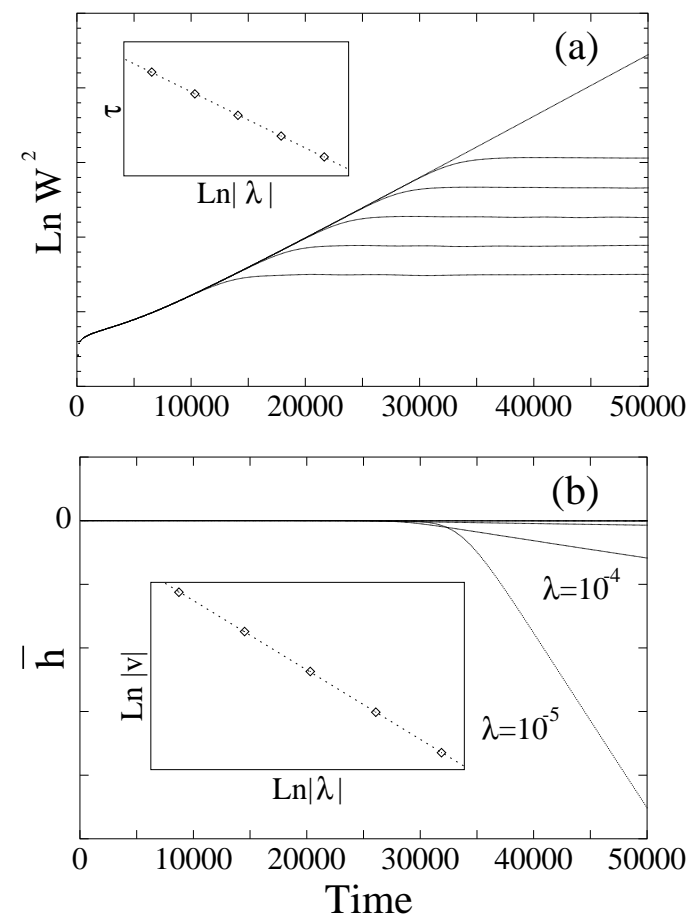

FIG. 1. Time evolution of (a) the surface width $W^{2}$ and (b) the mean height $\bar{h}$ for the parameters $\nu_{x}=-0.0001$, $\nu_{y}=-0.6169, K_{x}=K_{y}=K_{x y} / 2=2$. The different curves correspond to different values of $\lambda_{x}=\lambda_{y}=\lambda$. In (a), from top to bottom, the curves correspond to $\lambda=0,-10^{-5},-10^{-4}$, $-10^{-3},-10^{-2}$, and $-10^{-1}$, respectively. In (b), from bottom to top, they correspond to $\lambda=-10^{-5},-10^{-4},-10^{-3}$, $-10^{-2}$, and $-10^{-1}$, respectively. Inset (a): The crossover time $\tau$, estimated from (a) is shown as a function of $\ln |\lambda|$. Inset (b): Plot of $\ln |v|$ versus $\ln |\lambda|$. The dotted line has a slope $\approx-1.07$, implying $v \sim 1 / \lambda$.

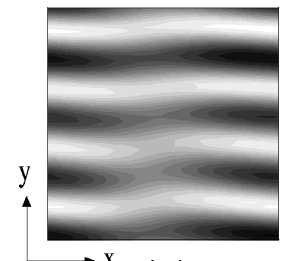

(a)

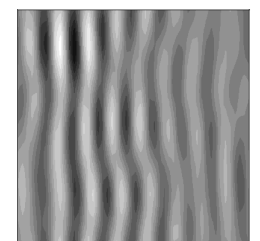

(b)

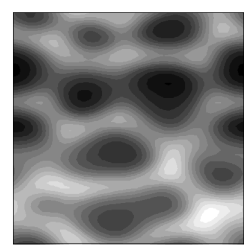

(c)
FIG. 2. Grey scale plot of a surface of size $64 \times 64$ with the parameter sets: (a) $\nu_{x}=-0.0001, \nu_{y}=-0.6169$, $K_{x}=K_{y}=K_{x y} / 2=2$, and $\lambda_{x}=\lambda_{y}=-0.001$ at $t=2 \times 10^{4}$. (b) $\nu_{x}=-1.2337, \nu_{y}=-0.0001, K_{x}=K_{y}=K_{x y} / 2=1$ and $\lambda_{x}=\lambda_{y}=-0.001$ at $t=2 \times 10^{3}$. Eq. (2) predicts the wavelength $\ell_{y} \approx 16$ for (a), and $\ell_{x} \approx 8$ for (b). (c) The surface configuration at time $t=5 \times 10^{5}$ for the parameter set used in (a).

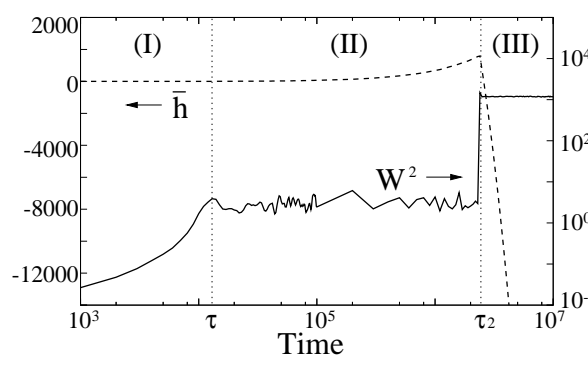

(a)

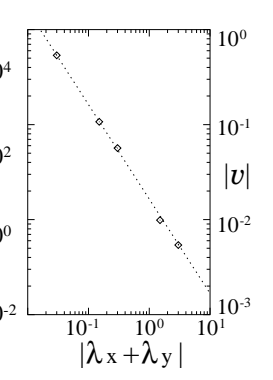

(b)
FIG. 3. (a) Time evolution of the mean height $h$ (dashed, left linear scale) and the surface width (solid, right logarithmic scale) for the parameters, $\nu_{x}=-0.6169, \nu_{y}=-0.01$, $K_{x}=K_{y}=K_{x y} / 2=2, \lambda_{x}=1$ and $\lambda_{y}=-4$. The dotted lines seperate the three regimes discussed in the text. (b) The dependence of $|v|$ on the nonlinear terms $\left|\lambda_{x}+\lambda_{y}\right|$ for the same parameters used in (a). The dotted line has a slope $\approx-1.02$, implying $v \sim 1 /\left(\lambda_{1}+\lambda_{2}\right)$.

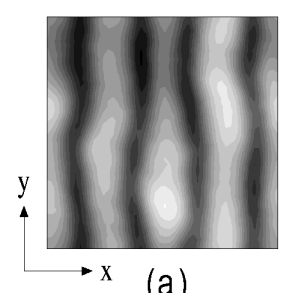

(a)

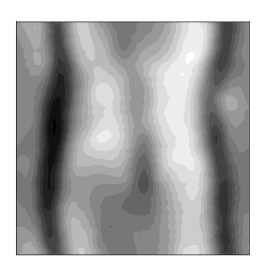

(b)

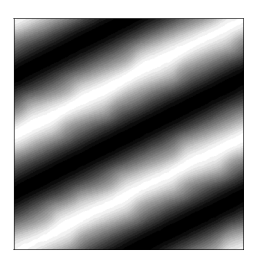

(c)
FIG. 4. Grey scale plot of a surface of size $64 \times 64$ with the parameter set used in Fig. 3, showing the surface morphologies at $t=10^{4}$ in regime $\mathrm{I}$ (a), $t=2 \times 10^{5}$ in regime II (b), and $t=10^{7}$ in regime III (c). 